\title{
Business transformation projects-virtual reality systems (VRS)
}

\author{
Antoine Trad \\ IBISTM, France \\ Damir Kalpić \\ University of Zagreb
}

Faculty of Electrical Engineering and Computing, Croatia

\section{Keywords}

Business transformation, virtual reality, Artificial Intelligence (AI), Education and sports, eActivity

\begin{abstract}
This article proposes the fundaments of Artificial Intelligence (AI) and is the basics to support Virtual Reality Systems (VRS) for Education and Sports (VRS4ES). The Applied Holistic Mathematical Model (AHMM) for AI (AHMM4AI) is the result of research on AI, business, education, digital sport, financial and organizational transformations using a mathematical model's concept. This research project is based on an authentic and proprietary mixed research method that is supported by an underlining mainly qualitative holistic reasoning model module that uses quantitative functions (Trad \& Kalpić, 2020a). The VRS4ES uses the Artificial Intelligence Pattern (AIP) to manage eActivity (eEducation or eSports) processes. Such projects are cross-functional and complex undertakings, developed using selection-based classification and weightings of critical success factors and areas, which are used as global variables in VRS4ES. In this article the main subject is VRS4ES that uses AIP for optimal integration purposes. Such transformation projects can be applied to various types of VRS, like team competition, personal training, intelligent support activities and other. People have become addicted to virtual environments and videogames since the 80s, but what is interesting, is the attractiveness they boast nowadays. eActivity, or competitive videogame playing, have exponentially expended in the last decade, to the level that in 2017 the second most watched sporting event in the USA, after the Superbowl, was an eActivity competition (Acer, 2018).
\end{abstract}

\section{Introduction}

The problem in designing the VRS4ES, is the starting point and it is a rational start to define intelligence (Merriam-Webster, 2020a):

The ability to learn or understand or to manage new or revisited situations, using skilled use of reason.

The ability to apply knowledge to manipulate environments or to think abstractly as measured by an objective criterion (such as experiments results). This sentence defines the basics of engineering.

$\mathrm{AI}$ is the ability of an automated and monitored team, organization or group (simply an Entity) to perform intelligent-cognitive tasks/scenarios and react flexibly to its environment in real-time, like in eActivity or video gaming. Such scenarios are used to maximize the chances or probability of achieving a particular goal. An AIP needs a global approach for VRS4ES using a Decision-Making System for AI (DMS4AI) that can integrate into an Entity, like an educational organization's processes or eActivity teams. AIP uses a Natural Language Programming for AI (NLP4AI) that can be adopted by the project's development teams for implementing an VRS4ES (Myers, Pane \& Ko, 2004). AIP based VRS4ES uses an Enterprise Architecture Integration for AI (EAI4AI) methodology as its skeleton.

\section{Background}

A societal, educational or business Transformation Project (simply the Project) that is based on a dynamic Information and Communication System (ICS) can support Entities to integrate complex VRS4ES strategies. The DMS4AI is used to solve VRS4ES requests or problems by offering a set of possible solutions in the form of actions and recommendations. The DMS4AI uses a central qualitative method based on a beam search (using a generic heuristic tree) that in turn uses targeted quantitative methods at its nodes. The DMS4AI supports the AIP based VRS4ES by using its multi-objective, multi-project, Critical 
Success Factor (CSF) mechanisms. The authors based their research using existing industry standards, like for example The Open Group's Architecture Framework's (TOGAF) and its Architecture Development Method (ADM) (The Open Group, 2011a). A transformation manager (or simply the Manager) can integrate an DMS4AI in the transformation roadmap to support its complex and risky VRS4ES' integration process (Zaiane \& Ben Moussa, 2018). The research's methodology is based on 1) a multidomain Literature Review Process (LRP); 2) a mixed qualitative and quantitative methodology; 3) VRS4ES major references and trends analyses; and 4) an engineering-oriented Proof of Concept (PoC) (or a controlled experiment), which is the optimal for AI based engineering projects (Easterbrook, Singer, Storey, \& Damian, 2008). The DMS4AI interacts with VRS analysts by means of an interface in order to manage the CSFs and to launch the reasoning process, where the Decision-making module (Dm) interacts with other modules. The research's main strength is the classification of CSF sets in Critical Success Areas (CSA) and their usage in the DMS4AI.

\section{The research and development project's structure for AI}

The main topic of the research is related to Projects, AI, VRS and their risk management capacities. The ultimate global research's Research Question (RQ) is: "Which transformation manager characteristics and which type of support should be assured in the implementation phase of a transformation project?". This article's RQ is: "What are the fundaments of AIP in the context of a VRS4ES?".

\section{The Research Basics}

The AIP based VRS4ES, and the actual article is a part of the Selection management, Architecturemodelling, Control-monitoring, Decision making, Training management, Project management, Finance management, Geopolitical management, Knowledge management and Implementation management Framework (SmAmCmDmTmPmFmGmKmImF, for simplification reasons, in further text instead of the term Transformation Research \& Architecture Development framework, just Environment will be used.

\section{The Mechanics of CSFs Evaluation}

The research for VRS4ES processing starts with the first phase (PHASE_1) called the feasibility phase, which checks if the whole Project and its AIP based VRS4ES approach, is feasible. Then tries to evaluate the success rate using the most important CSFs, using Environment's Dynamic Rules for AI (DR4AI). DR4AI artefacts are organised, parsed and weighted by the internal DMS4AI' engine. If PHASE_1 is satisfying and credible, then it is possible to move to the second phase (PHASE_2). Environment phases for a single research iteration. To implement this model there is need to understand the structure of a Holistic AIP implementation.

\section{Natural AI Predisposition Quantum's Support}

AIP's approach has an important impact on VRSs, and the way professionals prepare for competitions or exams. AIP automation can implement robots to prepare athletes in traditional sports. This holistic evolution permits to process data and allows athletes to prepare efficiently. Quantum for AI (Q4AI) is a cross-functional or a holistic field that is used for building quantum algorithms which can support computational tasks within AI fields like Machine Learning for AI (ML4AI). Q4AI has a holistic approach.

\section{A Holistic View}

Holism promotes a view presenting the whole as intimate strongly interconnected modules, which cannot exist independently in the end system, and they can be regarded as the sum of its modules. Holism is the opposite of a localized approach that creates isolated isles of knowledge. Holism is the approach where modules should be viewed as wholes of a unique system, not only a collection of ad-hoc algorithms, as it is often applied. The term Holism was coined by Jan Smuts in his 1926 book Holism and Evolution.

\section{The Brain as an AI Etalon}

Today, most scientists admit the fact that the brain is a complex combination of holism and phrenology, especially with the discovery of association cortices. Even, if holistic views are vastly 
predominant, it was noticed and accepted that some specific/localized cortical areas were necessary for sensory inputs, where the high order of processing was carried out by the cortex as a whole, or in a holistic approach. The more complex question is whether a holistic brain can be part of a more complex network of brains (Unam, 2020); such a network would correspond to the collaboration of eActivity teams.

\section{Collective Human Interaction-Electricity}

Direct brain-to-brain communication has been a subject of intense interest in the last decades, mainly driven by advanced AI initiatives. Merging and analysing of human brain activities is the future of human collective AI activities, like in eActivity teams. Conducted studies, in which researchers linked together brains of several rats, using complex implanted electrodes known as brain-to-brain interfaces; resulted in what is known as the first organic computer with living brains collaborating as if they were a set of coordinated microprocessors. The animals in this network learned to synchronize the electrical activity of their nerve cells to the same extent as those in a single brain. Networked brains were tested for things such as their ability to discriminate between two different patterns of electrical stimuli and they routinely outperformed individual animals (Martone, 2019). Another approach is the case of exoskeleton as the external AI based skeleton supporting and protecting a body, in contrast to the internal skeleton (endoskeleton). Larger kinds of exoskeletons are known as Shells (Wikipedia, 2020).

\section{AI Basics \\ Definitions}

The main definitions for $\mathrm{AI}$ are:

McKinsey defines AI, as the ability of a machine to perform advanced cognitive functions; where it associates it with human minds, like perceiving, reasoning, learning, interacting with humans and the environment, problem solving, and exercising creativity (Chui, Kamalnath, \& McCarthy, 2019).

European Commission defines AI, as a system that shows intelligent behaviour, by analysing their environment and that can perform various tasks with autonomy, in order to achieve specific goals (European Commission, 2019).

Stanford University defines AI, as science and engineering fields for making intelligent machines, especially intelligent software. It is software which tries to understand human intelligence, where AI does not have to limit itself to biologically observables activities (McCarthy, 1989).

Wikipedia defines AI as intelligence demonstrated by machines, in contrast to the natural intelligence, displayed by humans and animals (Wikipedia, 2020).

The Environment defines AI, as a generic and holistic systemic intelligence heuristics-based pattern.

\section{Approaches and Contradictions}

To analyse the various requirements of AIP based VRS, there is a need to understand the various existing contradictions and problems. And above all imagination, as Albert Einstein defines: Imagination is more important than knowledge. For knowledge is limited, whereas imagination embraces the entire world, stimulating progress, giving birth to evolution... (Einstein, \& Shaw, 2012). Nikola Tesla did not believe in logical thinking alone and he expressed that intuition and imagination can support transformational initiatives. The answer to the mentioned contradictions is to have strongly interconnected granular microartefacts which simulate neural elements.

\section{Common Constructs and Artefacts}

For all AI domains can be classified using the following constructs and artefacts:

LRP: where before starting an AI processing task, there is a need to verify the analysed subject and looking for any existing evidence (references) to be used. This is called PHASE_1.

CSFs: can map to any type of variable in any AI field, like for example a dependent or independent variable.

A tree: is a generic problem-solving tree can be applied to all the defined domains.

Goal Function for AI (GF4AI) output asserts if the expected result was met.

Rules and constraints define the CSFs (or variables) limitations and if violated, related actions are to be launched.

Iterations define, the maximum number and current iteration value. 
Data as an input, define the sets of input data.

Data as an output, defines the type of output data storage.

Processing success defines, the success conditions for each iteration.

Error coefficient defines the tolerance to be respected.

Data Science Integration for AI based and VRS4ES

Data Science Integration for AI (DSI4AI) main characteristics are (Le Baher, 2019):

The understanding of the integration of complex AI algorithms, which can be a hideous task. Nevertheless, it is useful to get insights for future analysis of various CSFs like performance indicators and other.

In a performance-oriented environment, the main goal is to maximize the CSF related to Project's success. Most of quantitative Key Performance Indicators (KPI) are correlated and are related to the selected CSFs, with a configurated kill participation and assists in achieving maximum values. Kill participation is the percentage of a team's hits in which a player participates.

The $1^{\text {st }}$ iteration concerns teams where players who achieved kills in organised/grouped competitions are better rewarded. It is observed that values are anti-correlated to the optimal value, where damage was done. KPIs could give hints about the metagame and could be studied in more in-depth.

Vision supports the role for farming, gold earned or damage outputs. First blood presence is slightly correlated to the win-rate and should not be significant CSF. It should not be considered as the main feature for futures DSI4AI based systems. First Blood is an in-game term, referring to the first kill in a game session.

\section{AHMM4AI AND COMMON CONSTRUCTS}

Common Constructs

Constraints and Rule based Engines for VRS

Constraints and DR4AI characteristics are:

AIP defines a set of assertions, stored in a database which maintains data about VRS' current state or knowledge.

Centralization in one data space, where the rule engines are optimized.

A rule set is a representation of an expert's knowledge and corresponds to a CSF.

The integration of a rule engine and a scripting engine in an AIP based VRS.

Critical Success Areas, Factors and Related Project Items

CSF for AI (CSF4AI) characteristics are:

The CSF4AI is based on CSAs.

A CSF corresponds to a microartefact scenario (a set of actions).

CSFs are important for the mapping between the AIP's resources, VRS requirements, microartefacts' scenarios and the VRS4ES platform.

The research starts with PHASE_1, to check whether the whole undertaking makes sense.

The Basic Nomenclature

The AHMM4AI has a defined nomenclature to facilitate AIP's usage needed to solve VRS problems. When using AHMM4AI for problem solving, starting with the tree's initial state that has a related GF4AI. The solution is the path through the tree state, where is given an initial/root state, and which satisfies the GF4AI (Tolos, 2018).

\section{Heuristics, ML4AI, Empirics and Action Research}

An AHMM4AI instance can launch a qualitative beam-search based heuristic processes (Della Croce \& T'kindt, 2002), where weightings and ratings concept support VRS requests. Actions Research (AR) (Berger, \& Rose, 2015) can be considered as a set of continuous beam-search heuristics processing phases and is synchronized with ADM's phases (Järvinen, 2007). Fast transformation requests may provoke an important set of events and problems that can be hard to predict and solve. The AHMM4AI is responsible for the qualitative heuristic process to support VRS4ES' problem solving and synchronizes a set of AHMM4AI instances which have also separate heuristics processes (Nijboer, Morin, Carmien, Koene, Leon, \& Hoffman, 2009). AR based heuristics enables reflective practice that is the basis of a holistic approach to develop VRS solutions DMS4AI (Leitch, \& Day, 2006). 


\section{The Applied Transformation Mathematical Model}

The generic AHMM can be applied to any domain. In this article, the Domain, is VRS (AHMM4AI = AHMM(AI)). The model of an AIP based Transformation Mathematical Model (GAIPTMM), abstracts the Project for a given Entity. The GF4AI of the GAIPTMM's formula can be optimized by using constraints and with extra variables that need to be tuned using the AHMM4AI. The variable for maximization or minimization can be, for example, can be the Project success (Sankaralingam, Ferris, Nowatzki, Estan, Wood \& Vaish, 2013). The GAIPTMM is the combination of AIP based VRS, Project and the ADM.

\section{The applied case study for aip based VRS4ES}

ICS and EAI4AI Infrastructure

The PoC uses a set a Case Study (CS), which includes:

An eActivity game from the official competition of FIFA in Football, that has been produced by EA SPORTS for over 20 years and is now the largest sports video game.

A specialized technical infrastructure transformations case (Jonkers, Band \& Quartel, 2012a), developed by the Open Group and a set of other cases like Kinect gaming kit, they offer the capability to implement the AIP.

Concerning AIP based development, the needed goals to be achieved are:

The DMS4AI delivers a possible set of solutions.

Select a set of objectives from the proposed set of CSAs.

Builds AI microartefacts to support the various types of AIP based scenarios.

Prepare Environment's PHASE_1 and if successful, select a problem from the CS to prove research's PHASE_2.

Integrating CSFs

A CSF and its KPI enumerations are measurable and mapped to a weighting that is roughly estimated in the first iteration and then tuned through ADM iterations, to support the VRS. A set of CSFs are essential. The main issue is how to define the AIP's main goals to integrate the DMS4AI and how to interrelate the different fields like VRS fields.

THE ICS CSA

A Holistic Microartefacts Implementation Model for AIP

The AIP microartefact model includes (Daellenbach \& McNickle, 2005):

Actual ICS methodologies/architecture, modelling and development enclose various automated NLP4AI microartefacts.

NLP4AI microartefacts' management contain micro-actions that manage various AIP activities.

The AHMM4AI structure is used to orchestrate NLP4AI microartefact instances and receive/evaluate change requests.

The AIP is based on a holistic systemic approach to use all the Environment's NLP4AI micro artefacts.

\section{ADM'S INTEGRATION PROCESSES}

The AIP supports the mapping and modelling of micro artefacts to VRS4ES' activities. The VRS is based on the EAI4AI (and other) standards and frameworks to align micro artefacts with existing ADM.

The Unit of Work-An Extreme Granular Approach

Defining AIP micro artefacts' granularity and responsibility for a Project is a complex process; added to that, there is the complexity in implementing the "1:1" mapping and classification of the discovered VRS micro artefacts. The AIP uses standard design methodologies like the TOGAF's ADM. The AIP's design and mapping concepts are supported by a set of the Environment's components where its internal NLP4AI implements micro artefacts to evaluate expressions, according to the AHMM4AI principles (Neumann, 2002).

\section{The holistic strategic human resources system for AIP}

Concerning the Human Factors (HR) CSA, in the authors previous research works, concluded that a manager is Architect of Adaptive Business Information Systems (AofABIS), specialized in the implementation phase of a Project (Trad \& Kalpić, 2020a).

Critical Success Areas and Factors for AIP 
The sets of HRS CSFs are presented in the form of a real-world constraints, which affect VRS' activities. Managers for VRS' integration might benefit from this research, while its ambition is to be considered as a major Project managerial benefit.

Needed HR Skills for the Selected Profile's Skills

There is no concrete educational curriculum for a Manager for VRS integration topics, where bookkeeping and political sciences profiles are chosen for such tasks. There is an essential need for more research on VRS Managers' profiles and their educational prerequisites. The needed skills must comprise the knowledge of VRS's: 1) AIP processes; 2) automated process management; 3) agile Project management; 4) team behaviour; 5) AI; 6) VRS development kits; and 7) ICS' and VRS' implementation skills. It is recommended to use the AofABIS profile for VRS' integration.

Educational Requirements

Managers need hands-on skills and educational curriculum that includes the following set of skills: 1) VRS architectures and processes management; 2) complex VRS integration initiatives; 3) agile AIP based development; 4) KMS4AI management and integration; 5) VRS' design; 6) DMS4AI management and AI methodologies; 7) VRS4ES functional domains.

The Role of Education

Basic Education Constraints

The role of VRS4ES in education is described as follows (Acer, 2018):

eActivity has expended in the last decade. Because of its growth, Entities should consider this its impact on future generations.

Higher education needs eActivity that have already gained its place in higher education systems worldwide.

Like traditional athletes, successful videogame players in high schools are awarded with scholarships for their studies. The main motivation for this trend is the proven impact of high videogaming skills on Science, Technology, Engineering...

The value of eActivity in education can make confusions between serious educational approach and mere entertainment.

There are initiatives to convince parents about eActivity in the educational system, where their children will experience video gaming in an educational environment. Such an approach can make students more prepared for the future.

A strong relation between eActivity and engineering subjects has been already proven. Competitive video gaming can enhance the development of engineering skills ...

The Evolution of Behaviour

VRS communities are heavily suffering from toxic behaviours, especially in competitions. There can generate negative behaviour, such as harassment and making barriers for players achieving high performance, to reduce players' efficiencies in order to make them leave the game. Sources indicate that the health players are at stake, they do not face identical challenges regarding a sane and healthy lifestyle as the average population. The transformation game training and integrating it in the real world, is a way to integrate physical activity and other behaviours in training (Rudolf, Bickmann, Froböse, Tholl, Wechsler, \& Grieben, 2020).

\section{Holistic decision and knowledge making systems}

AI Basic Structure

The Use of Heuristic Trees

A Heuristic Decision Tree (HDT) is an abstract model and can be defined as a collection of nodes linked together through edges in a hierarchical structure. They have no cyclic relations and there is only one path to a particular node (Open4Tech, 2019). There are different types of HDTs, like Binary Tree, Binary Search Tree, Red-Black tree, AVL tree, Heap, etc. The deciding factor on which tree to use, is mainly performance. Since HDTs are data structures, performance is measured by inserting and retrieving data.

Evaluating the Decision

HDT's evaluation starts from the root node to the right-hand side and moves to the left; and the main steps are:

Conference proceedings of the Centre for Business \& Economic Research, ICBED-2021, 10-12 June 
All nodes are labelled, and it starts with the closest node to the right-hand side, labelling the top and then the bottom nodes.

Then, recursively moving from right to left, weighting VRS4ES results.

Finally, the optimal actions are offered, based on the option that gives the best results.

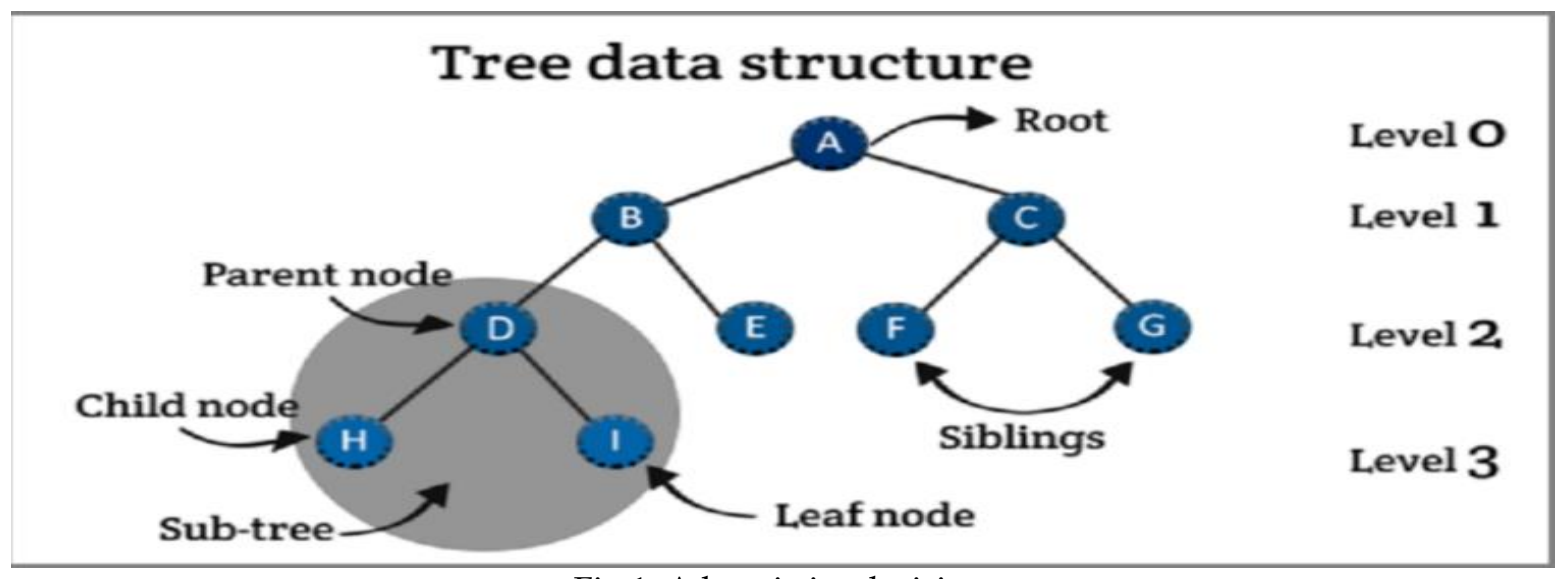

Fig 1. A heuristics decision tree.

\section{Complex Decision based Systems}

DMS4AI supports VRS4ES requests, which are processed by using an AHMM4AI's instance, that in turn is based on the selected CSAs and CSFs. The DMS4AI and KMS4AI have a complex system nature.

Rule based Engine for AI

The VRS4ES uses rules based DMS4AI and its main characteristics are the following (Krasner, 2020):

It is rule-based and covers most of the so-called ML4AI.

There are various manners to integrate VRS where data play a major role, and the quality of data is the most important CSF.

Best practices can be used to choose the right AIP methods.

ML4AI can be used to store human knowledge into DMS4AI as rules.

The DR4AI is optimal, but many rules can limit VRS' capabilities.

The Use of Machine Learning for VRS

The DMS4AI uses ML4AI, where its main characteristics are (Krasner, 2020):

DR4AI is a static intelligence approach, in contrast with ML4AI that is an adaptive approach.

ML4AI has the ability to deduce new rules and to eliminate the rules that are not useful.

ML4AI learns in various ways, but supervised training is generally the first step in an ML4AI process.

ML4AI interprets, classifies and performs tasks/scenarios using unstructured data.

To define VRS's integration strategy and AIP is essential.

Knowledge Management System for AI

This research relates and assembles AIP micro artefacts and resources. AIP micro artefacts are linked with the KMS4AI (The Open Group, 2011a). The Project identifies the sets of CSAs and CSFs for the KMS4AI and DMS4AI.

The DMS4AI and VRS4ES

The DMS4AI, where the user configures the NLP4AI micro artefacts and CSFs. These NLP4AI micro artefacts are orchestrated by the AHMM4AI choreography engine. The DMS4AI' actions map to the AIP which delivers actions. The AHMM4AI formalism is implemented in VRS' actions; such a set of actions can be modelled and managed by the AHMM4AI that is implemented with an experiment or a PoC (The Open Group, 2011a). The main impacts of DMS4AI on VRS4ES are (Bailey, 2012):

Rare research initiatives have researched the relationship between complex DMS4AI usages and the VRS domain.

Results show that some types of VRS domains increase aggression and affective processing. 
These effects are relevant given the impact of executive functioning and affective processing on the quality of VRS.

DMS4AI is a crucial part of successfully achieving VRS' goals, including the VRS4ES.

The negative association between VRS and cognitive control because time spent, which is a period for the development.

\section{The Role of The Vrs Platform}

Technological Fundaments

Basic Technologies

The platforms' fundaments are (Alton, 2019):

eActivity is the forefront of VRS4ES, where technology plays a crucial role.

Goldman Sachs notes that: To play a traditional sport, one typically needs access to an appropriate venue (field, court, arena, etc.), and to be successful, it almost always helps to be big, fast, strong, or coordinated.

Building eActivity infrastructure needs: 1) Agile networks; and 2) On-Premises and edge technology.

Network speed, agility and bandwidth are critical CSFs, and one of the biggest issues that affects eActivity space is latency.

Cloud companies are focused on building network stability and reducing latency.

On-Premises solutions for security, since activities are online; on-premises technology is preferred for eActivity.

Basic specs are provided by the competition leagues and offer a preview on tech needs evolution.

Asset tagging helps in tracking of devices and managing their setup.

Monitors trace the visual experience where monitors are used during gameplay, practice and competitions.

Accessories like keyboards to headsets have an important role for gaming experience.

Cameras help to experience the thrill of eActivity.

Services for tuning eActivity components keeps the platform on point, is an important part of staying competitive.

Using Rules Engines

VRS4ES is complex that includes Internet of Things (IoT) and the the following rules engines (Waylay, 2020):

Rule's engines are based on forward chaining algorithms, knowing that most of the IoT based platforms use rules engines.

Condition/action rules engines are supporting VRS, even though they are using single conditional statement.

Stream processing rules engines process data in motion, directly as they are produced.

Event processing engines are the predecessors of stream engines and differ from them in the way they handle events.

HDT are a concise visual representation for specifying which actions to perform, depending on given conditions.

Trends

VRS platforms' main trends are (Verdict, 2020):

eActivity is the fastest growing gaming domain, this multiplayer VRS has a spectacular growth over the last decade.

eActivity is expanding and in VRS events thousands of spectators are filling stadiums to participate in live events.

VR4AI, using headsets and new games, is a fresh market for VR4AI eActivity that is emerging.

AIP is an integral stub for eActivity and includes ML4AI training platforms for skills and strategy development.

DSI4AI, extracts insights from data used by Entities across VRSs.

VRS4ES' Robustness

AI is evolving, disruptive in impact and pervasive in presence, its significant computational capabilities and vast potential for deep learning applicable to many areas. Together with 5G connectivity, the IoT, and the Cloud, AI is becoming transformative technologies, giving new opportunities. AI is used 
automotive, manufacturing, healthcare and in defence industries, with datacentre and cloud computing infrastructure forming the principal areas in which AI training is done.

The Role of Big Data Integration for AIP

The role Big Data Integration for AI (BGI4AI) characteristics are (Wooden, 2021):

BGI4AI analytics and cloud technologies are used to improve performance.

VRS teams develop strategies, while operators want to provide enhanced viewing experiences and better regulations.

BGI4AI supports VRS where success is often determined by strategy and the analysis of past performance.

It is possible to collect statistical info on matches from websites, but the capacity to have deep analysis is not a simple task.

Tools for measuring are evolving and VRS4ES can simulate a team and process huge amounts of data.

VRS advancing, with potential for providing intelligence for marketing, professional performance and regulation.

Cloud infrastructure and BGI4AI analytics are integrated in VRSs.

THE IMPLEMENTATION'S POC

The CS

The CS is related to two cases, which includes an insurance claims system (CS_1) and an VRS gaming case (CS_2), where the AIP is the VRS4ES' main interface.

The PoC

The CS_1 Phase

The used CSFs has bindings to research resources, where the AIP was designed using an NLP4AI microartefacts. In this article's tables and the result of the processing of the DMS4AI, as illustrated in

Table 1, show clearly that the AIP is not an independent component.

\begin{tabular}{|c|c|c|c|c|c|}
\hline & Critical Success Factors & \multicolumn{2}{|c|}{ KPIs } & Weightings Ranges & Values \\
\hline 1 & RDP4AI & Feasible & $\tau$ & From 1 to 10. & 9,5 \\
\hline 2 & AHMM4AI & Feasible & 7 & From 1 to 10 . & 9,6 \\
\hline 3 & ACS4AI & Feasible & - & From 1 to 10. & 9,25 \\
\hline 4 & ICS & Feasible & - & From 1 to 10. & 9,75 \\
\hline 5 & ADM4AI & Proven & - & From 1 to 10 . & 10 \\
\hline 6 & HR4AI & Feasible & 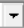 & From 1 to 10 . & 9,4 \\
\hline 7 & KMS4AI & Feasible & - & From 1 to 10. & 9,25 \\
\hline 8 & DMS4AI & Feasible & - & From 1 to 10 . & 9,2 \\
\hline 9 & PLATFORM & Complex & - & From 1 to 10. & 8,4 \\
\hline
\end{tabular}

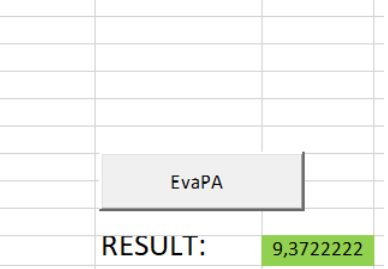

Table 1. The research's overall outcome is (rounded) 9,40

The Environment and hence AIP's main constraint is that CSAs for simple research components, having an average result below 8.5 will be ignored. In the case of the VRS4ES' implementation, an average result below 6.5 will be ignored. AIP supports the relationships between the VRS4ES, this research's requirements, NLP4AI generic and micro artefacts, unique identifiers and the CSAs.

\section{The CS_2 Phase-The Data Platform Setup}

With CS_1's ending, CS_2 starts, with adapting BGI4AI to the data modelling part and it concerns the CS. ArchiSurance data architecture describes the relationships between the conceptual business objects and the logical data objects. ArchiMate also defines the ICS's Information Structure viewpoint, which is similar to the traditional data models. The used data viewpoints that the CS uses is the Logical Data diagram; that shows a subset of the business objects that ArchiSurance defines (Jonkers, Band \& Quartel, 2012a).

A component, the customer data, is in the insurance file, which contains: 1) insurance requests for VRSs; 2) insurance policies for VRSs; and 3) damage claims. The purpose of the Data Dissemination diagram is to show the relationship between the entity sets data, business services, and application components. The diagram shows how the logical objects are to be implemented. Assigning VRS requirements to data is a CSF that is related to the competition criticality of the VRS infrastructure (Jonkers, Band, \& Quartel, 2012).

\section{The CS_2 Phase-Using AIP's HDT}


Like mentioned previously, the FIFA environment was installed, in which all teams' activities were stored in the form of logs. Log records are scanned and mapped to the CSF and HDT models, by using the Environment. From the Environment client's interface, the NLP4AI interface was launched. Then the NLP4AI implements the needed micro artefact scripts to process the defined CSAs. These scripts make up the AIP's set of actions. The HDT is applied on a specific CSF that is mapped to a specific VRS problem and a set of actions, like for example the VRS' central log server's resources overflow problem. This problem was selected as using the log files of the VRS. HDT's algorithm uses DSI4AI calls to quantitative methods and delivers the VRS team recommendation on how to react.

\section{Conclusion}

Table 1 showed that VRS4ES' implementation is a complex but not a risky process except for the resources' limits and the PoC has achieved the objectives. The VRS4ES is mapped to the Project's processes and the aggregation of all the CSA tables exceeds the defined minimum, then the PoC was used for problem solving using the HDT with calls to quantitative methods. This article uses a mixed action research model, where CSFs support VRS specialists prepare sessions, and the main recommendations are:

Project has sub-projects, where the priority is to transform their DMS4AI, KMS4AI and the existing platform.

A distributed DMS4AI must be built to support Managers on various levels to manage AIP based VRS4ES.

Existing methodologies improve the robustness of VRS's infrastructure, which replaces the ancient infrastructure.

A VRS has to build an ICS, to counter fraud and ensure eActivity team's success, like the ones organized by major financial institutions can be expected (Stupples, Sazonov, \& Woolley, 2019).

VRS4ES supports the strategy and preparing eActivity teams.

\section{References}

Acer (2018). Education Trends-How Education can benefit from eActivity. Acer.

https://acerforeducation.acer.com/education-trends/how-education-can-benefit-from-eActivity/, accessed on 18FEB-2021.

Alton, L. (2019). An Introductory Guide to eActivity Technology. Connected. https://community.connection.com/anintroductory-guide-to-eActivity-technology/

Bailey, K. (2012). What would my avatar do? Video games and risky decision making. Graduate Theses and Dissertations Iowa State University Capstones, Theses and Dissertations. Iowa State University. USA.

Berger, J., \& Rose, J. (2015). Nine Challenges for e-Government Action Researchers. International Journal of Electronic Government Research.

Cearley, D., Walker, M., \& Burke, B. (2016). Top 10 Strategic Technology Trends for 2017.

Daellenbach, H., McNickle, D. \& Dye, Sh, (2012). Management Science - Decision making through systems thinking. 2nd edition. Palgrave Macmillian. USA.

Della Croce, F., \& T'kindt, V. (2002). A Recovering Beam Search algorithm for the one-machine dynamic total completion time scheduling problem, Journal of the Operational Research Society, 53:11, 1275-1280. Taylor \& Francis.

De Ambroggi, L. (2019). Artificial intelligence will be transformative across industry domains and verticals. IHS MArkit.

Easterbrook, S., Singer, J., Storey, M., \& Damian, D. (2008). Guide to Advanced Empirical Software Engineering-Selecting Empirical Methods for Software Engineering Research. F. Shull et al. (eds.). Springer.

Einstein, A., \& Shaw, G; (2012). Cosmic religion, With other opinions and aphorisms. Dover Publications.

Gunasekare, U. (2015). Mixed Research Method as the Third Research Paradigm: A Literature Review. Volume 4 Issue 8, August 2015. University of Kelaniya. IJSR.

Järvinen, P. (2007). Action Research is Similar to Design Science. Quality \& Quantity. February 2007, Volume 41, Issue 1, pp 37-54. Springer Verlag. Germany. Retrieved August 10, 2018, from

https://link.springer.com/article/10.1007/s11135-005-5427-1

Jonkers, H., Band, I., \& Quartel, D. (2012a). ArchiSurance Case Study. The Open Group.

Krasner, E. (2020). How to choose between rule-based AI and machine learning. TechTalks.

Le Baher, H. (2019). Data Science as support of eActivity performance and strategies (I)-First step : A general case study about the League of Legends World Championship. Towards data science. 
Leitch, R. \& Day, Ch. (2000). Action research and reflective practice: towards a holistic view. Taylor \& Francis. Queen's University of Belfast, United Kingdom. Retrieved February 10, 2018, from

https:// www.tandfonline.com/doi/ref/10.1080/09650790000200108?scroll=top

Martone, R. (2019). NEUROSCIENCE: Scientists Demonstrate Direct Brain-to-Brain Communication in Humans. SCIENTIFIC AMERICAN, A DIVISION OF SPRINGER NATURE AMERICA, INC.

https://www.scientificamerican.com/article/scientists-demonstrate-direct-brain-to-brain-communication-inhumans/

Merriam-Webster (2020a). intelligence. Intelligence | Definition of Intelligence by Merriam-Webster.

Myers, B., Pane, J. \& Ko, A. (2004). Natural programming languages and environments. ACM New York, NY, USA.

Neumann, G. (2002). Programming Languages in Artificial Intelligence. Bidgoli (ed) Encyclopaedia of Information Systems, Academic Press, San Diego, CA, ISBN 0-12-227240-4, 2002, pages 31-45.

Nijboer, F., Morin, F., Carmien, S., Koene, R., Leon, E., \& Hoffman, U. (2009). Affective brain-computer interfaces: Psychophysiological markers of emotion in healthy persons and in persons with amyotrophic lateral sclerosis. 3rd International Conference on Affective Computing and Intelligent Interaction and Workshops. IEEE.

Open4Tech (2019). Trees vs. Graphs. Open4Tech. https://open4tech.com/trees-vs-graphs/

Potier, A. (2020). NATURAL LANGUAGE PROCESSING: HOW AI UNDERSTANDS OUR LANGUAGES. Konverso.

Rudolf, K., Bickmann, P., Froböse, I., Tholl, Ch., Wechsler, K., \& Grieben, Ch. (2020). Demographics and Health Behavior of Video Game and eActivity Players in Germany: The eActivity Study 2019. National Center for Biotechnology Information, U.S. National Library of Medicine. Rockville Pike, Bethesda. USA. https://www.ncbi.nlm.nih.gov/pmc/articles/PMC7142975/

The Open Group (2011a). Architecture Development Method. The Open Group. USA. Reviewed in February 2018, http://pubs.opengroup.org/architecture/togaf9-doc/arch/chap05.html.

Sankaralingam, K., Ferris, M., Nowatzki, T., Estan, C., Wood, D., \& Vaish, N. (2013). Optimization and Mathematical Modeling in Computer Architecture. Morgan \& Claypool Publishers.

Stupples, B., \& Sazonov, A., \& Woolley, S. (2019). UBS Whistle-Blower Hunts Trillions Hidden in Treasure Isles. Bloomberg-Economics. Bloomberg. Reviewed in November 2019

https://www.bloomberg.com/news/articles/2019-07-26/ubs-whistle-blower-hunts-trillions-hidden-in-treasureislands

Tolos, J. (2018). Artificial intelligence-An introduction to AI. Meduim. https://medium.com/@joantolos/artificialintelligence-abe8f1b1cfc2

Trad, A. \& Kalpić, D. (2017d). A Neural Networks Portable and Agnostic Implementation TKMEF for Business Transformation Projects- The Basic Structure. IEEE Conference on Computational Intelligence. France.

Trad, A \& Kalpić, D. (2019e). Business Transformation and Enterprise Architecture-The Holistic Project Resources Management Pattern (HPRMP). Encyclopaedia, IGI-Global. USA.

Trad, A., \& Kalpić, D. (2020a). Using Applied Mathematical Models for Business Transformation. IGI Complete Author Book. IGI Global.

Zaiane, S. \& Ben Moussa, F. (2018). Cognitive Biases, Risk Perception, and Individual's Decision to Start a New Venture. International Journal of Service Science, Management, Engineering, and Technology (IJSSMET). IGI Global. DOI: 10.4018/IJSSMET.2018070102.

Unam (2020). COGNITIVE FUNCTIONS. Unam. https://www.ifc.unam.mx/Brain/speech.htm

Violino, B. (2018). Designing and building AI infrastructure. Techtarget.

https://searchenterpriseai.techtarget.com/feature/Designing-and-building-artificial-intelligence-infrastructure

Verdict (2020). Analysis-eActivity: Technology Trends GlobalData Thematic Research. Verdict. https://www.verdict.co.uk/eActivity-technology-trends/

Wikipedia (2020). Business rules engine. Wikipedia the free encyclopaedia.

Wooden, A. (2021). How BGI4AI is Revolutionising. The Future of eActivity. Intel Corporation. 